
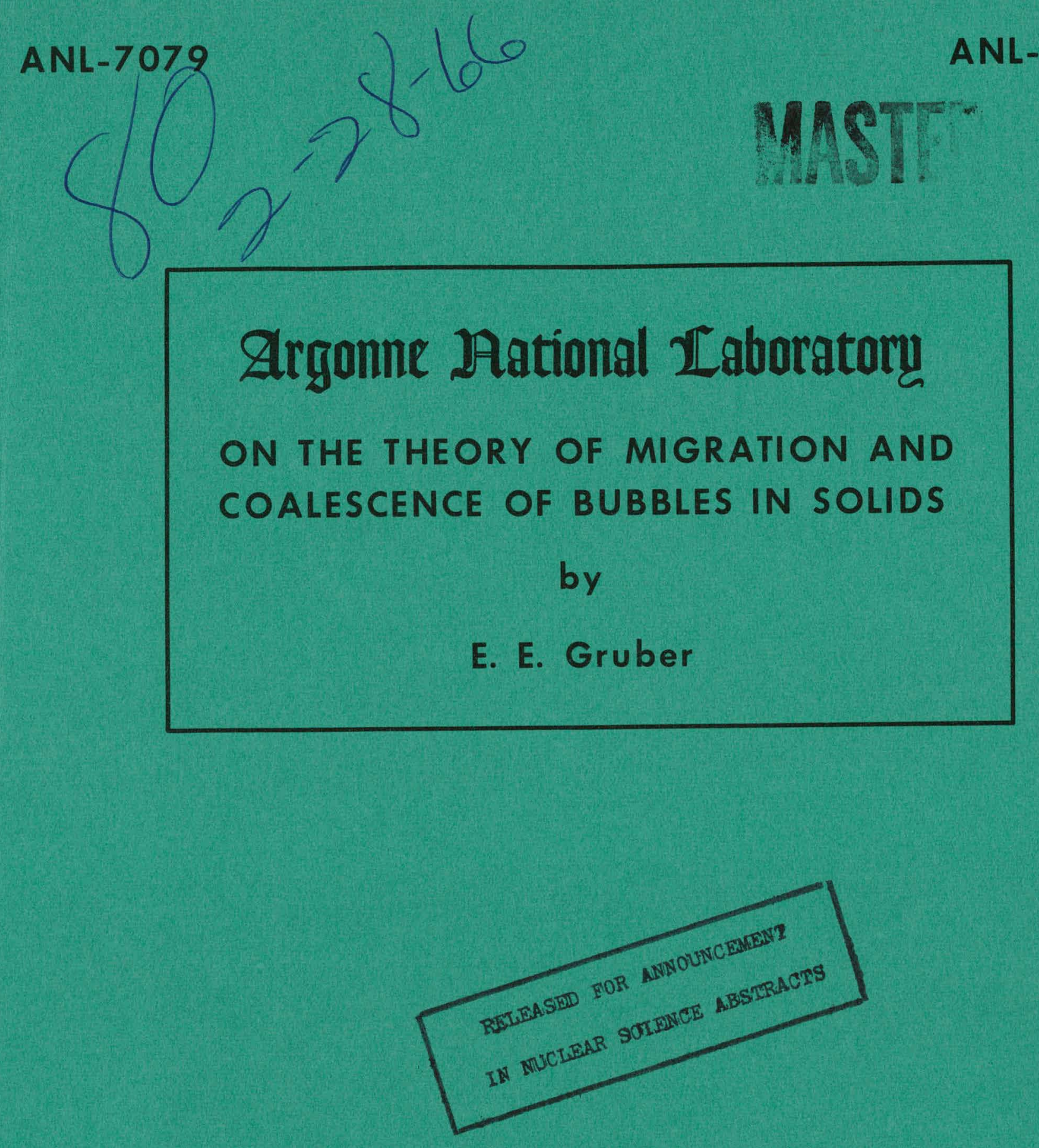


\section{DISCLAIMER}

This report was prepared as an account of work sponsored by an agency of the United States Government. Neither the United States Government nor any agency Thereof, nor any of their employees, makes any warranty, express or implied, or assumes any legal liability or responsibility for the accuracy, completeness, or usefulness of any information, apparatus, product, or process disclosed, or represents that its use would not infringe privately owned rights. Reference herein to any specific commercial product, process, or service by trade name, trademark, manufacturer, or otherwise does not necessarily constitute or imply its endorsement, recommendation, or favoring by the United States Government or any agency thereof. The views and opinions of authors expressed herein do not necessarily state or reflect those of the United States Government or any agency thereof. 


\section{DISCLAIMER}

Portions of this document may be illegible in electronic image products. Images are produced from the best available original document. 


\section{LEGAL NOTICE}

This report was prepared as an account of Government sponsored work. Neither the United States, nor the Commission, nor any person acting on behalf of the Commission:

A. Makes any warranty or representation, expressed or implied, with respect to the accuracy, completeness, or usefulness of the information contained in this report, or that the use of any information, apparatus, method, or process disclosed in this report may not infringe privately owned rights; or

B. Assumes any liabilities with respect to the use of, or for damages resulting from the use of any information, apparatus, method, or process disclosed in this report.

As used in the above, "person acting on behalf of the Commission" includes any employee or contractor of the Commission, or employee of such contractor, to the extent that such employee or contractor of the Commission, or employee of such contractor prepares, disseminates, or provides access to, any information pursuant to his employment or contract with the Commission, or his employment with such contractor.

Printed in USA. Price $\$ 1.00$. Available from the Clearinghouse for Federal Scientific and Technical Information, National Bureau of Standards, U. S. Department of Commerce, Springfield, Virginia 
ANL-7079

Metals, Ceramics, and Materials

(TID-4500, 47th Ed.)

AEC Research and

Development Report

ARGONNE NATIONAL LABORATORY

9.700 South Cass Avenue

Argonne, Illinois 60439

ON THE THEORY OF MIGRATION AND

COALESCENCE OF BUBBLES IN SOLIDS

by

E. E. Gruber

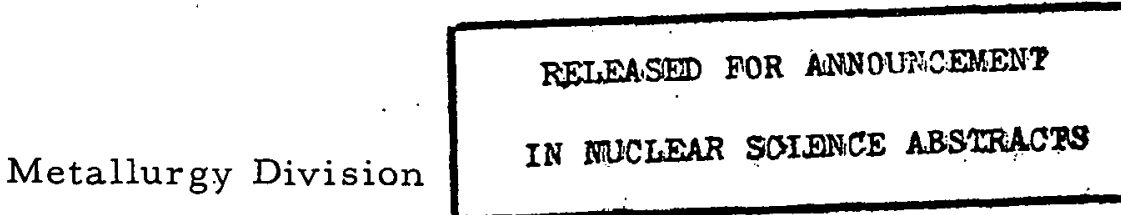

Program 4.10.38

Portions of the material in this report have appeared in the following Metallurgy Division Annual Reports:

$$
\begin{aligned}
& \text { ANL-6868 (1963), pp. } 399-408 \\
& \text { ANL-7000 (1964), pp. } 237-239
\end{aligned}
$$

November 1965

Operated by The University of Chicago

under

Contract W-31-109-eng-38

with the

U. S. Atomic Energy Commission 
TABLE OF CONTENTS

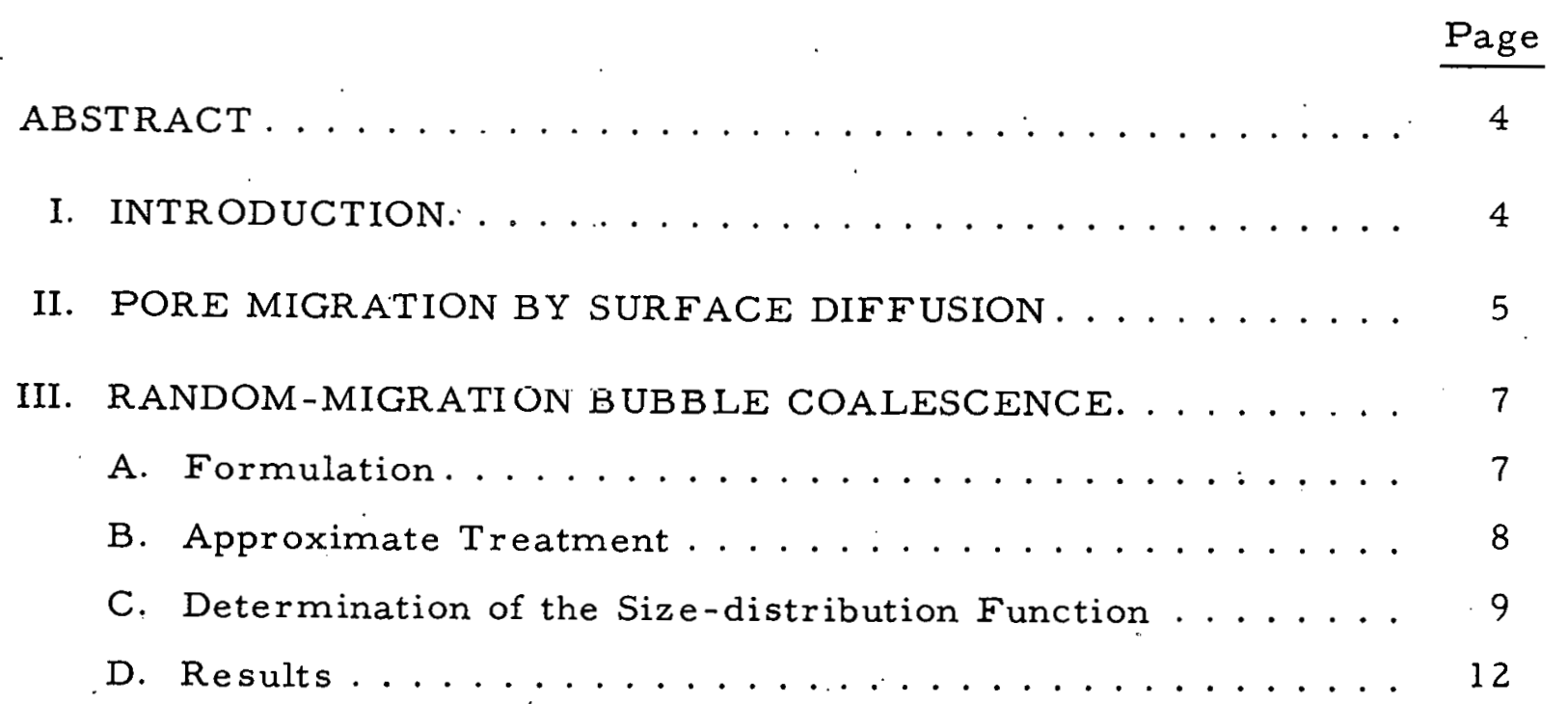

IV. BIASED-MIGRATION BUBBLE COALESCENCE. . . . . . . . 14

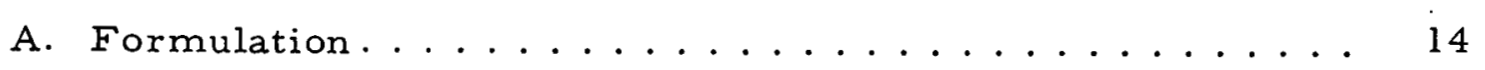

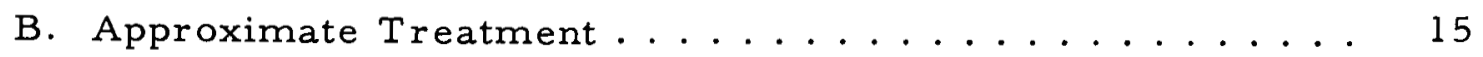

C. Determination of the Size-distribution Function ...... 16

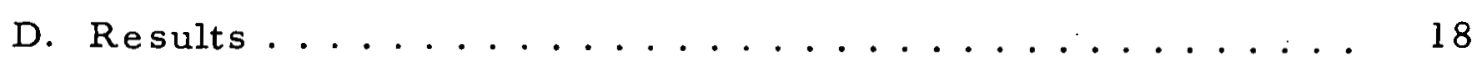

V. DISCUSSION OF RESULTS ....................... 20

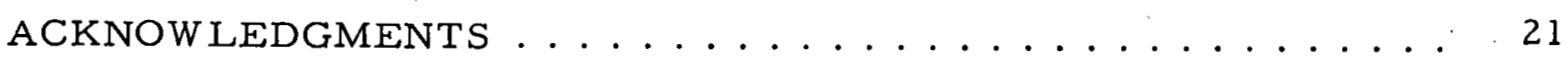

REFERENCES .......................... 22 


\section{LIST OF FIGURES}

No.

Title

$\underline{\text { Page }}$

1. Schematic Representation of a Migrating Pore in a Solid....

2. Size-distribution Functions at Different. Times for Random-

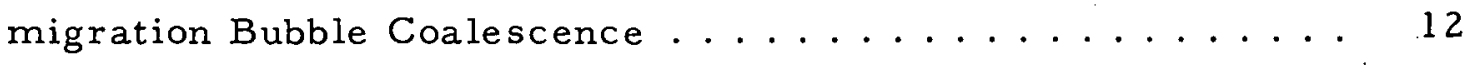

3. Dependence of Bubble Distribution Parameters on Time .... 13

4. Size-distribution Function for Random Coalescence . . . . . 13

5. Size-distribution Functions at Different Times for Biasedmigration Bubble Coalescence............... 17

6. Semilogarithmic Plot of Distribution Function for Biased-

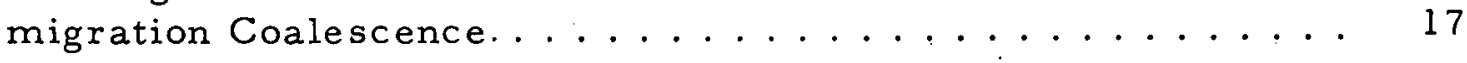

7. Dependence of Bubble-distribution Parameters on Time.... 18

8. "Standardized" Size-distribution Curves for Biased-migration

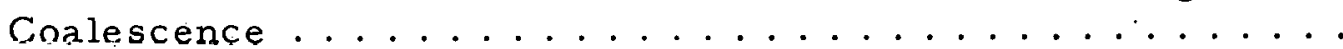




\title{
ON THE THEORY OF MIGRATION AND \\ COALESCENCE OF BUBBLES IN SOLIDS
}

by

E. E. Gruber

\begin{abstract}
The surface-diffusion migration of an isolated pore in a solid is analyzed in detail. The results, which are similar to earlier results, are applied in analyses of bubble coalescence during postirradiation annealing. Coalescence is considered, first, as a result of random migration of bubbles, in which case the results confirm earlier results that the mean bubble radius should be proportional to (time $)^{1 / 5}$, and second, as a result of biased migration of bubbles. In the latter case, the predicted mean radius increases linearly with time. In both cases, the bubble-size distribution is calculated and used to predict the swelling of the solid, which is about $15 \%$ greater in the first case and up to $65 \%$ greater in the second case than predicted by approximate treatments based on change in the mean bubble radius with time.
\end{abstract}

\section{INTRODUCTION}

Considerable interest has been shown recently in the subject of gasfilled pores, or "bubbles," in solids. Although interest developed initially because of the swelling of nuclear fuel materials due to the presence of entrapped fission gases, increasing consideration is being given to bubbles as a means of studying certain basic properties of materials. This interest has been spurred by the demonstration by Barnes and Mazey that helium bubbles can be observed in alpha-particle irradiated copper foils upon pulse heating in the electron microscope. Valuable information concerning the anisotropy of surface tension of solids under a very pure inert atmosphere can be obtained by observation of bubble shapes, which should be related to the equilibrium shape of a fixed volume of solid. The feasibility of this approach has been demonstrated by Nelson, Mazey, and Barnes. ${ }^{2}$

The present work is concerned with the relationship between surface diffusion and bubble migration and coalescence. Shewmon ${ }^{3}$ has shown that the observation by Barnes and $\mathrm{Mazey}^{1}$ that small bubbles migrate in a thermal gradient with a velocity inversely proportional to the bubble radius indicates that surface diffusion is the dominant transport mechanism, so that 
a small bubble moves primarily by diffusion of metal atoms over the inner surface of the bubble. Shewmon has suggested the possibility of studying the rate of spheroidization of two bubbles that coalesce, in conjunction with the analysis of Nichols, ${ }^{4}$ as a means of determining the surface-diffusion coefficient. Beçause of the rapid approach to the equilibrium shape for small bubbles, the surface-diffusion coefficient $D_{s}$ should be measurable at lower temperatures and on more materials than otherwise possible. ${ }^{3}$

The approach in the present analysis is to consider the migration and coalescence that result under certain idealized situations in the hope that experimental observations of bubble migration and coalescence under laboratory conditions can give useful information concerning the surfacediffusion coefficient and other material parameters. It is assumed throughout that the gas behaves ideally, that the gas pressure is balanced by surface tension, and that re-solution of the gas does not occur. Because of the negligibly small importance of very small bubbles with regard to swelling, deviations from the above-assumed behavior should not be a significant source of error. It is further assumed that the gas is initially present as very small bubbles (so that nucleation is ignored), and that coalescence occur $\bar{s}$ instantaneously upon collision of two bubbles. Finally, it is assumed for the present analysis that the matrix is free of dislocations and grain boundaries. and that bubbles do not interact except upon collision.

\section{PORE MIGRATION BY SURFACE DIFFUSION}

Since coalescence is regarded in this analysis as essentially a collision process, the relations for pore migration are basic to the analysis of coalescence. The velocity of a spherical pore migrating by surface diffusion will first be calculated by considering

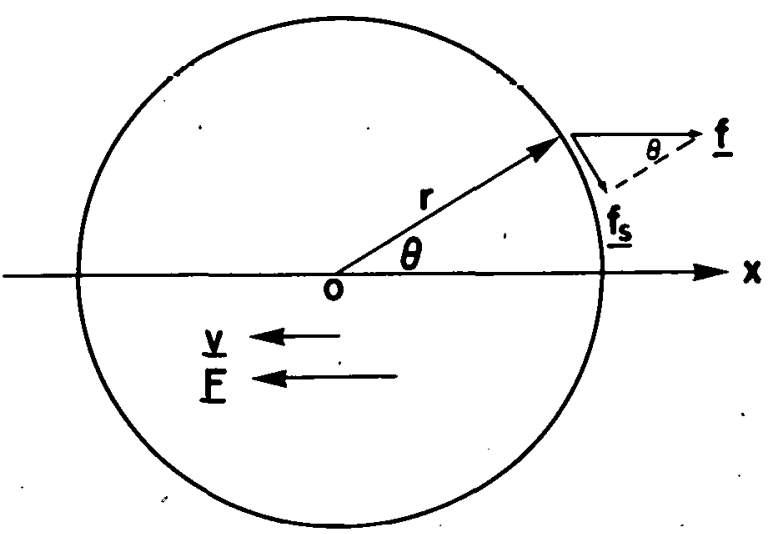

39587

Fig. 1. Schematic Representation of a Migrating Pore in a Solid the driving force as a force on the individual atoms comprising the pore surface. This furce per atom will then be related to the equivalent force on the pore, after which the results will be used to define a pore-diffusion coefficient, $\mathrm{D}_{\mathrm{b}}$.

It is assumed that the pore is initially spherical with radius $r$ and is centered at the origin, as shown in Fig. l. If a force $\underline{f}$ is exerted in the positive $\mathrm{x}$-direction on each mobile atom in the surface, the pore will migrate in the opposite direction with 
a velocity $\underline{v}$, as though the pore were driven by an equivalent force $\underline{F}$. The flux $j_{s}$ across a unit length on the surface is given by the product of the surface density $\nu$ of atoms involved in surface diffusion and the average drift velocity $V$, which is given by the Nernst-Einstein relation. ${ }^{5}$ This flux is

$$
\mathrm{j}_{\mathrm{S}}=\nu \mathrm{V}=\nu \frac{\mathrm{D}_{\mathrm{s}}}{\mathrm{kT}} \mathrm{f}_{\mathrm{S}}=\frac{\nu \mathrm{D}_{\mathrm{S}}}{\mathrm{kT}} \mathrm{f} \sin \theta
$$

where $D_{\delta}$ is the surface-diffusion coefficient, $k T$ has the usual meaning, and $f_{S}=f \sin \theta$ is the magnitude of the component of the force $\underline{f}$ paraliel to the surface.

The rate at which a surface element moves normal to the surface is equal to the rate of volume change per unit area; this change is given by the product of atomic volume $\Omega$ and the negative divergence of the flux. The unit of area is taken as a.strip of surface at constant $\theta$ of width $r \Delta \theta$, because of the rotational symmetry about the $x$-axis. The flux leaving this strip by crossing the line at $\theta$ is given by the product of flux per unit length and the line length,

$$
j_{-}=\frac{2 \pi r \nu D_{s} f}{k T} \sin ^{2} \theta \text {. }
$$

The flux entering the strip at $\theta+\Delta \theta$ is

$$
j_{+}=\frac{2 \pi r \nu D_{s}^{f}}{k T} \sin ^{2}(\theta+\Delta \theta)
$$

Since the area of the strip is $2 \pi r^{2} \sin \theta \Delta \theta$ for small $\Delta \theta$, the speed of a surface element normal to the surface is

$$
\frac{d \rho}{d t}=\lim _{\Delta \theta \rightarrow 0} \frac{\left(j_{-}-j_{+}\right) \Omega}{2 \pi r^{2} \sin \theta \Delta \theta}=-\frac{\Omega \nu D_{s} f}{r k T \sin \theta} \frac{d\left(\sin ^{2} \theta\right)}{d \theta},
$$

where $\mathrm{d} \rho / \mathrm{dt}$ is the time rate of change in length of the vector from the origin. Substituting for the derivative and taking $\Omega \nu=\Omega^{1 / 3}=0.89$ la for the fcc lattice, where $a_{0}$ is the interatomic distance, we obtain

$$
\frac{\mathrm{d} \rho}{\mathrm{dt}}=-\frac{1.78 \mathrm{a}_{0} \mathrm{D}_{\mathrm{s}} f}{\mathrm{rkT}} \cos \theta
$$

It can be shown from this equation that the change in $\rho$ with $t$ is such that the pore remains spherical and translates along the $x$-axis. The translation velocity can be obtained by setting $\theta=0$ in Equation (1) to give

$$
v-\frac{1.78 \mathrm{a}_{0} \mathrm{D}_{\mathrm{s}}}{\mathrm{rkT}} \mathrm{f}
$$


This result is in close agreement with the result obtained by Greenwood and Speight ${ }^{6}$ by a different and somewhat approximate treatment, and is equivalent to the expression obtained by $S_{\text {Shewon }}{ }^{3}$ by an alternate, approximate treatment.

The atomic driving force $f$ can be related to the equivalent force $F$ on the pore by considering the work done by the force $\underline{F}$ in moving the pore a distance $l$. This work ( $F l$, where $F$ is the magnitude of the force $F$ ) is equivalent to the work done by the force $\underline{f}$ in moving an equivalent number $\left(\frac{4}{3} \pi \mathrm{r}: / \Omega\right)$ of atoms a distance $\ell$ in the opposite direction. It follows that

$$
f=-\left(3 \Omega / 4 \pi r^{3}\right) F
$$

where the minus sign is included because the forces act in opposite directions. For the fcc lattice, $\Omega=a_{0}^{3} / \sqrt{2}$, so that substitution for $f$ from Equation (3) into Equation (2) gives:

$$
\mathrm{v}=\frac{0.301 \mathrm{a}_{0}^{4} \mathrm{D}_{\mathrm{S}}}{\mathrm{r}^{4} \mathrm{kT}} \mathrm{F} \equiv \frac{\mathrm{D}_{\mathrm{b}}}{\mathrm{kT}} \mathrm{F}
$$

The Nernst-Einstein relation has been used in the last step to define an equivalent volume-diffusion coefficient $D_{b}$ for the surface-diffusion migration of the bubble,

$$
\mathrm{D}_{\mathrm{b}} \equiv 0.301 \mathrm{D}_{\mathrm{s}}\left(\mathrm{a}_{0} / \mathrm{r}\right)^{4}
$$

This relation differs only slightly in the numerical coefficient from the relation obtained by Greenwood and Speight ${ }^{6}$ by an alternate method.

\section{RANDOM-MIGRATION BUBBLE COALESCENCE}

\section{A. Formulation}

It is assumed in the present case that coalescence follows bubble collisions resulting from random migration of bubbles in an infinite, perfect crystal. A close analogy exists between this problem and that of colloid coagulation as treated by Chandrasekhar, ${ }^{7}$ who gives for the number of collisions $\Delta F_{i j}$ of colloid particles in time $\Delta t$

$$
\Delta F_{i j}=4 \pi D_{i j} R_{i j} F_{i} F_{j}\left[1+\frac{R_{i j}}{\left(\pi D_{i j} t\right)^{1 / 2}}\right] \Delta t .
$$

In the case of bubble coalescence, $\Delta F_{i j}$ is the number of coalescences between $i$ and $j$ bubbles in time $\Delta t$, where it is convenient to associate $i$ and $j$ with particular values of $n_{i}$, the number of gas atoms in a bubble 
of radius $r_{i}$. D $D_{i j}$ is the appropriate diffusion coefficient, shown by Chandrasekhar to be given by $D_{i}+D_{j}, R_{i j}$ is the sum of the bubble radii $r_{i}+r_{j}$, and $F_{i}$ represents the concentration (number per unit volume) of bubbles containing $n_{i}$ gas atoms each. If the sum of the radii of the bubbles in question is smaller than the mean distance travelled by the two bubbles relative to one another, the second term in the brackets in Equation (6) can be neglected (as was done by Chandrasekhar). Equation (6) can then be written in terms of the definition of $\mathrm{D}_{\mathrm{b}}$ [Equation (5)],

$$
\Delta F_{i j}=1.204 \pi a_{j}^{4} D_{s} F_{i} \Gamma_{j}\left(r_{i}+r_{j}\right)\left(r_{i}^{-4}+r_{j}^{-4}\right) \Delta t
$$

\section{B. Approximate Treatment}

It will be seen that the bubble-size distribution function $F\left(n_{i}, t\right)$ can be obtained from Equation (7) by the use of finite-difference methods with the electronic digital computer. It will be informative, however, to consider first an approximate solution. If the bubble-size distribution is characterized by a simplified distribution of $\mathrm{N}$ bubbles, each with radius $r_{i}$, Equation (7) can be simplified by substituting for $r_{j}=r_{i}$ and $F_{i}=F_{j}=N$. Then the decrease in $F_{i}$, the total number of bubbles that contain $n_{i}$ gas atoms each in time $\Delta t$, is given by

$$
\Delta F=2 \Delta F_{i i}=9.632 \pi a_{n}^{4} D_{S} N^{2} r_{i}^{-3} \Delta t
$$

since two bubbles disappear with each collision.

Barnes has shown that, according to our assumptions, $r_{i}$ is related to $\mathrm{n}_{\mathrm{i}}$ by the expression ${ }^{8}$

$$
r_{i}^{2}=(3 k T / 8 \pi \gamma) n_{i},
$$

where $\gamma$ is the surface tension of the solid. Since each bubble formed by coalescence contains $2 n_{i}$ gas atoms, it follows that the radius of each newly formed bubble is $\sqrt{2}: r_{i}$. For a total concentration of $m$ gas atoms per unit volume, the number of bubbles of radius $r_{i}$ before coalescence is

$$
F_{i}=\frac{m}{n_{i}}=\frac{3 m k T}{8 \pi \gamma r_{i}^{2}}
$$

where Equation (9) has been used to substitute for $n_{i}$ in terms of $r_{i}$. A direct calculation of the new mean radius after $\Delta F_{i i}$ collisions gives for small $\Delta \mathrm{F}_{\mathrm{i} i}$ the change in mean radius,

$$
\Delta r=\frac{1}{2}(\sqrt{2}-1)\left(\Delta F / F_{i}\right) r_{i} \text {. }
$$


Substitution for $\triangle F$ from Equation (8) and for $F_{i}$ from Equation (10) gives the result; upon passing to the limit and integrating from $r=r_{0} \approx 0$ at $t \stackrel{0}{=}$ to $r$ at $t$,

$$
r^{5}=3.74 \mathrm{mkTa}{ }_{0}^{4} \mathrm{D}_{\mathrm{s}} \mathrm{t} / \gamma
$$

For comparison, Speight ${ }^{9}$ obtained the result

$$
r^{5}=r_{0}^{5}+2.6 a^{6} \nu_{0} \text { fzbkTt. } \exp \left(-Q_{S} / k T\right) / \pi \gamma \Omega
$$

by summation of a geometric series of pairwise coalescence times. In this equation, $a$ is the mean atomic spacing, $\nu_{0}$ is the Debye frequency, $Q_{S}$ is the activation energy for surface diffusion, $f$ is an entropy factor involved in the diffusion jump, $b$ is the atomic concentration of gas in the sample, $z$ is a numerical factor $(\sim 4)$, and the remaining symbols were defined earlier. Upon substitution for $D_{s}=\frac{1}{4} a_{0}^{2} \nu_{0} f \exp \left(-Q_{s} / k T\right), a=\Omega^{1 / 3}$, and $m=b / \Omega$, and with the approximation that $r_{0}$ is negligible with respect to $r$, this equation reduces to

$$
r^{5}=6.6 m k T a_{0}^{4} D_{s} t / \gamma
$$

which differs from Equation (12) only in the numerical constant. The result given by Equation (12) is therefore not new. However, it provides a convenient means of considering one of the most critical objections to this type of solution, the use of a mean radius in characterizing the distribution function.

C. Determination of the Size-distribution Function

The mean radius is defined in terms of the distribution function by

$$
r(t)=\sum_{i=1}^{\infty} r_{i} F\left(n_{i}, t\right) / \sum_{i=1}^{\infty} F\left(n_{i}, t\right),
$$

or

$$
r(t)=\left(\frac{3 k T}{8 \pi \gamma}\right)^{1 / 2} \frac{\left\langle n^{1 / 2}\right\rangle}{\left\langle n^{0}\right\rangle}
$$

where Equation (9) has been used to substitute for $r_{i}$, and the moment of order $k$ at time $t$ is defined by

$$
\left\langle n^{k}\right\rangle=\sum_{i=1}^{\infty} n_{i}^{k} F\left(n_{i}, t\right) .
$$


The volume increase, or swelling, relative to unit initial volume is given by

$$
\Delta V=\frac{4}{3} \pi \sum_{i=1}^{\infty} r_{i}^{3} F\left(n_{i}, t\right)=0.173(k T / \gamma)^{3 / 2}\left\langle n^{3 / 2}\right\rangle,
$$

where, again, Equations (9) and (14) have been used to express the results in terms of the appropriate moment of the distribution function. These moments can be accurately calculated only if the entire distribition function. is known.

The distribution function $F(n, t)$ can be obtained from Equation (7) by digital-computer calculations based on finite-difference methods. Substitution for $r_{i}$ from Equation (9) in Equation (7) gives

$$
\Delta F_{i j}=91.7 a_{0}^{4} D_{s} F_{i} F_{j}(\gamma / k T)^{3 / 2}\left(n_{i}^{1 / 2}+n_{j}^{1 / 2}\right)\left(n_{i}^{-2}+n_{j}^{-2}\right) \Delta t .
$$

It is preferable to normalize the function $F(n, t)$ by dividing each value $F_{i}$ by $m$, so that the resulting function $f(n, t)$, which represents the number of bubbles per gas atom per unit volume, is independent of the concentration $m$. The result can be written

$$
\Delta f_{i j}=f_{i} f_{j}\left(n_{i}^{1 / 2}+n_{j}^{1 / 2}\right)\left(n_{i}^{-2}+n_{j}^{-2}\right) \Delta \tau,
$$

where $\tau$ is a dimensionless parameter, which we shall term the "reduced time," defined by

$$
\tau \equiv 91.7 \mathrm{a}_{0}^{4} \mathrm{D}_{\mathrm{s}} \mathrm{m}(\gamma / \mathrm{kT})^{3 / 2} \mathrm{t} .
$$

Equation (17) forms a convenient basis for the finite-difference calculations because the roulte are not explicit. functions of the various parameters.

In principle, the finite-difference method is used to calculate the change in an assumed distribution function $f(n, 0)$ in a very short "time step" $\Delta T$. This change is used to determine the new distribution, and the procedure is repeated until the desired number of time steps have been completed. The reduced time $\tau$ is given by the sum of the time steps. The assumed distribution in this case was such that all bubbles were initially monatomic; liat is,

$$
\begin{aligned}
& f\left(n_{i}, 0\right)=1 \text { for } i=1, \\
& =0 \text { for } i>1 \text {. }
\end{aligned}
$$


Since each collision results in the disappearance of one $i$ and one $j$ bubble and the formation of one $(i+j)$ bubble, the distribution at reduced time $\tau+\Delta \tau$ is found from the relations

$$
\begin{aligned}
& f\left(n_{i}, \tau+\Delta \tau\right)=f\left(n_{i}, \tau\right)-\sum_{j=i}^{\infty} \Delta f_{i j} ; \\
& f\left(n_{j}, \tau+\Delta \tau\right)=f\left(n_{j}, \tau\right)-\sum_{i=1}^{j-1} \Delta f_{i, j} ; \\
& f\left(n_{i+j}, \tau+\Delta \tau\right)=f\left(n_{i+j}, \tau\right)+\sum_{i, j}^{\prime} \Delta f_{i j} .
\end{aligned}
$$

The limits have been chosen to prevent double counting, and the sum in Equation (19c) is over all values of $i$ and $j$ such that $i \leq j$ and $n_{i+j}=$ $\mathrm{n}_{\mathrm{i}}+\mathrm{n}_{\mathrm{j}}$.

The method of using these relations in a digital-computer program was rclatively straightforward; 200 values of. $f_{i}$ were stored in memory and comparisons were made according to Equation (17) to calculate the change $\Delta f_{i j} / \Delta \tau$. The calculated changes were stored in memory for each value of $i$, and a value of $\Delta \tau$ was calculated such that the largest change $\Delta f_{i}$ would be some predetermined fraction of the largest value of $f_{i}$. This fraction was taken in most cases as 0.05 , which gave a reasonable compromise between the excessive computer time required for a smaller fraction and the excessive inaccuracy that would result for a larger fraction. The calculated value of $\Delta T$ was added to the time memory and was used to calculate the actual shanges $\Delta f_{i}$, which were then added to the previous values of $f_{i}$. The calculations were repeated until no further information could be gained. The total number of steps used was over 400, with a resulting range in $\tau$ of more than six powers of ten. A doubling procedure was used to increase the range of $n$ while retaining only 200 values of $i$ in memory; that is; $n_{i}=i$ was used until $f_{200}$ became significant; then $n_{i}=2 i$ was used, and the doubling of the increment $\Delta n$ was repeated as necessary.

After each set of five time steps, the distribution function was printed out and several calculations were made. The important results to be obtained from the calculations are the mean bubble radius $r$ and the relative swelling of the sample $\Delta \mathrm{V}$ as functions of the reduced time $\tau$. These parameters are defined in terms of moments of the function $F(n, t)$ by Equations (13) and (15). The first moment $\left\langle n^{1}\right\rangle$ is also of interest as a check on the accuracy of the calculation, since $\left\langle n^{1}\right\rangle$ is the total number of gas atoms per unit initial volume and should be independent of time and equal to $m$. The $\mathrm{k}^{\text {th }}$ moment, defined by Equation (14), is given in terms of the computer results by the approximate expression 


$$
\left\langle n^{k}\right\rangle=m \Delta n \sum_{i=1}^{200} n_{i}^{k} f_{i} .
$$

The following characteristic values of $\mathrm{n}$ were also calculated:

$$
\begin{aligned}
& \left.\mathrm{n}_{1}=\overline{\left(\mathrm{n}^{1 / 2}\right.}\right)^{2}=\left(\left\langle\mathrm{n}^{1 / 2}\right\rangle /\left\langle\mathrm{n}^{0}\right\rangle\right)^{2} ; \\
& \mathrm{n}_{2}=\overline{\mathrm{n}}=\left\langle\mathrm{n}^{1}\right\rangle /\left\langle\mathrm{n}^{0}\right\rangle ; \\
& \mathrm{n}_{3}=\left(\overline{\mathrm{n}^{3 / 2}}\right)^{2 / 3}=\left(\left\langle\mathrm{n}^{3 / 2}\right\rangle /\left\langle\mathrm{n}^{0}\right\rangle\right)^{2 / 3} .
\end{aligned}
$$

These characteristic values give the appropriate values of $\mathrm{n}$ to be used in calculation of the mean radius, the mean number of atoms per bubble, and the mean bubble volume. The differences in these values give an indication of the error inheren't in the earlier approximate treatments, which must assume all characteristic values to be the same. The rates of change of these values with $\tau$ were also calculated.

D. Results

As $T$ increases, it is expected that the coalescence of smaller bubbles to form larger ones will cause the distribution function to diminish with time

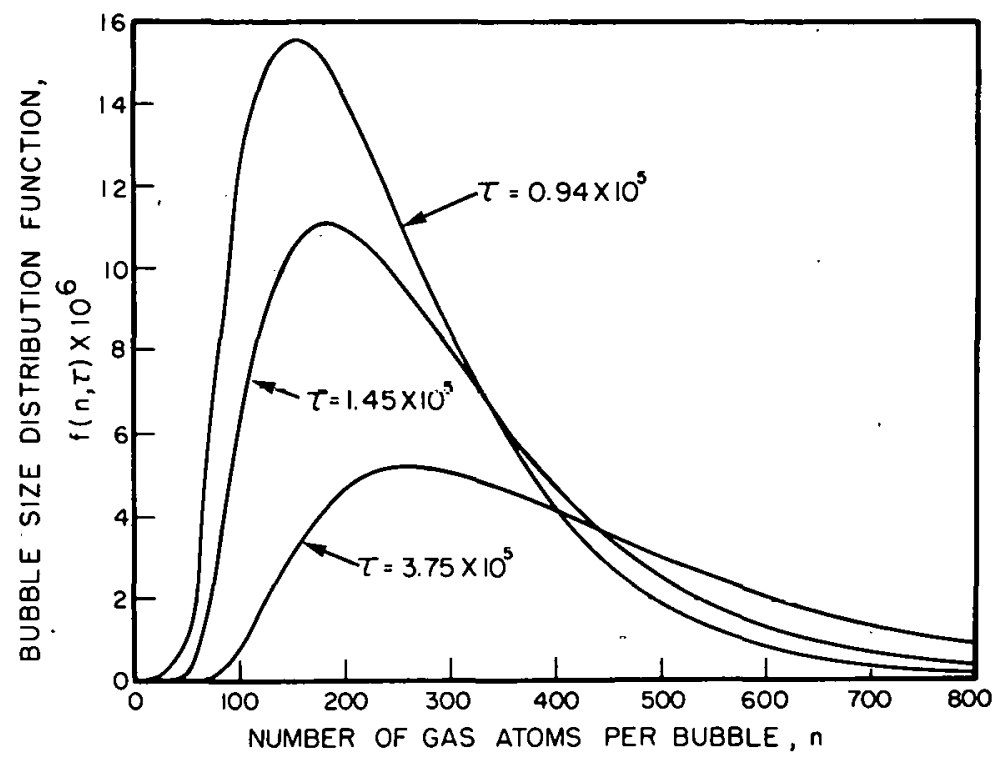

40412 for small $\mathrm{n}$ and to increase with time for large $n$. The area under the curve should also decrease, since the total number of bubbles decreases with time. This expected be havior was observed, as shown in Fig. 2 by the calculated functions for three values of $\tau$. The calculated distribution in each case was fairly smooth, although some oscillation was evident; $f_{i}$ was slightly greater for even values of $i$ than for odd values. However, this small oscillation did not significantly influence the results, as shown by the fact that after 400 time steps the calculated total number of gas atoms had dropped only to $0.99998 \mathrm{~m}$. 
Plots of the various parameters and successive distribution curves showed that after a very short time the shape of the distribution changed in a definite manner and that $\log -\log$ plots of the parameters as functions of $\tau$ yielded straight lines, as indicated in Fig. 3. The calculated slopes of the se plots varied within about $0.5 \%$ of $2 / 5$ for $n_{1}, n_{2}$, and $n_{3}$. The same accuracy was found for the other slopes, which were about $-2 / 5$ for $\left\langle n^{0}\right\rangle$, $-1 / 5$ for $\left\langle n^{1 / 2}\right\rangle, 0$ for $\left\langle n^{1}\right\rangle$, and $1 / 5$ for $\left\langle n^{3 / 2}\right\rangle$.

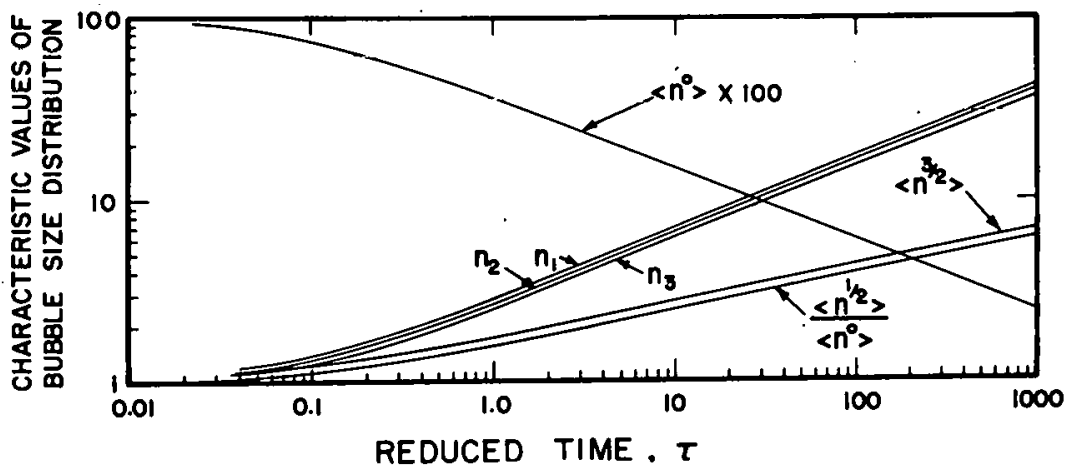

40449

Fig. 3. Dependence of Bubble Distribution Parameters on Time

The same results would be obtained if the distribution function were given by

$$
F(n, t)=m \tau^{-4 / 5} Z_{1}(u),
$$

where $u=n \tau^{-2 / 5}$. It is thus possible to standardize the distribution curve as shown in Fig. 4. The theoretical distribution function $F(n, t)$ can be calculated for any set of experimental parameters from this curve by multiplying the values on the ordinate by $\mathrm{m}^{-4 / 5}$ and those on the abscissa by $\tau^{2 / 5}$.

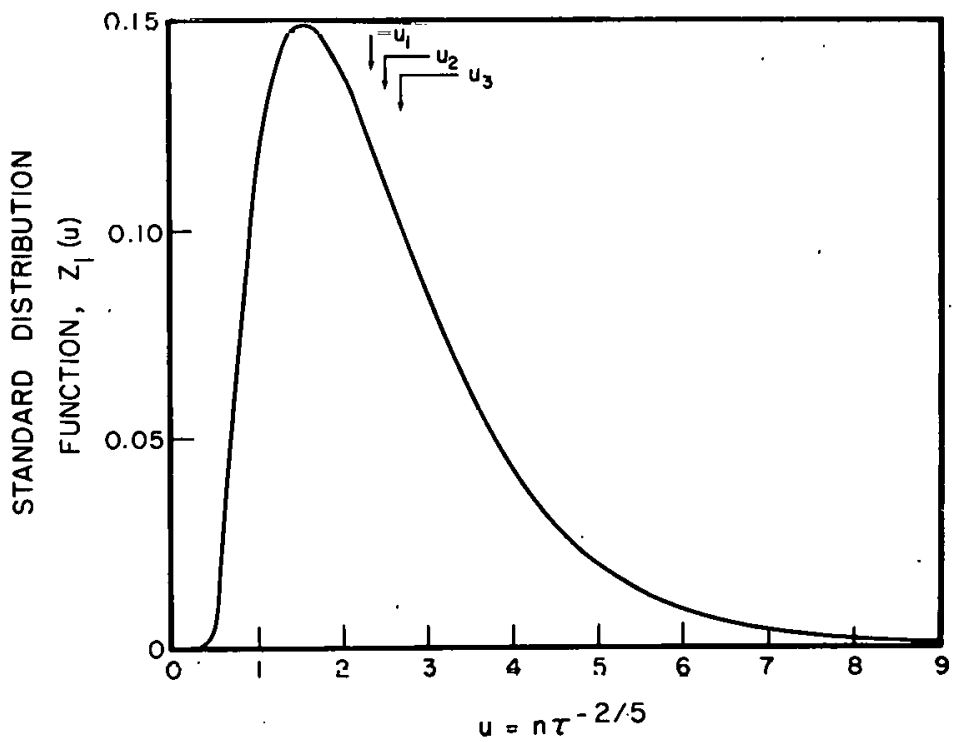

Fig. 4

Size-distribution Function for Raudum Coalescence 
The parameters can be expressed from these results as functions of $\tau$, and by substitution for $\tau$ from Equation (18), it can be shown that

$$
\mathrm{r}=1.30\left[\mathrm{mkTa}_{0}^{4} \mathrm{D}_{\mathrm{s}} \mathrm{t} / \gamma\right]^{1 / 5},
$$

and

$$
\Delta \mathrm{V}=0.75(\mathrm{mkT} / \gamma)\left[\mathrm{mkTa}{ }_{0}^{4} \mathrm{D}_{\mathrm{s}} \mathrm{t} / \gamma\right]^{1 / 5}
$$

As an example of the results predicted by this analysis, consider the case of copper at $1000^{\circ} \mathrm{K}$ with $\mathrm{m}=10^{20}$ helium atoms per $\mathrm{cm}^{3}, \gamma=1.7 \mathrm{x}$ $10^{3}$ ergs $/ \mathrm{cm}^{2}$, a $=2.55 \AA$, and $D_{s}=10^{-5} \mathrm{~cm}^{2} / \mathrm{sec}$. The term in brackets is $3.3 \times 10^{-42} \mathrm{t}$ ( $\mathrm{t}$ in seconds). For $\mathrm{t}=3 \times 10^{6} \mathrm{sec}$, or about one month, the predicted values are $\mathrm{r} \cong 42 \AA$ and $\Delta \mathrm{V} \cong 0.006 \%$.

The limited effect of random coalescence emphasizes the need for more quantitative consideration of other mechanisms of swelling, such as grain-boundary sweeping, ${ }^{10,11}$ dislocation effects, ${ }^{8}$ thermal gradients and other external effects that lead to unidirectional forces on bubbles, and perhaps bubble interactions.

Although the computer approach to the problem is not of great value in this particular case, the results clearly show that it is a valid approach to the problem. Further, it can be shown that the approximate solution gives results identical to Equations $(23 a)$ and (23b) except that the numerical coefficient for $\Delta \mathrm{V}$ is reduced to 0.65 . The error in $\Delta \mathrm{V}$ due to the use of. the approximate solution is about $15 \%$ in this case and can be much worse in other cases. Approximate treatments are more tenuous for the case of a uniform driving force, and some other approach such as that used here is necessary for a quantitative, theoretical treatment. This problem is considered in the next section.

\section{BIASED-MIGRATION BUBBLE COALESCENCE}

\section{A. Formulation}

If a uniform driving force that tends to move all bubbles in the same direction is present, it is not possible to characterize the distribution of bubble sizes by a mean radius $r$, since then all bubbles would migrate with the same speed and in the same direction, and no coalescence beyond that considered in the preceding section would occur. It is therefore necessary to consider the manner in which the bubble size varies in the sample. Coalescence occurs when a smaller bubble overtakes a larger, and hence slower, bubble. The collision volume is the volume of a cylinder of radius $r_{1}+r_{2}$ and of length $\left(v_{1}-v_{2}\right) \Delta t$, since only the difference in speed. leads to collisions. The probability of collision of a particular small bubble containing $n_{i}$ gas atoms with a larger bubble containing $n_{j}$ gas atoms is given 
by the product of collision volume and number of $n_{j}$ bubbles per unit volume, for a sufficiently small collision volume, so that the totalnumber of collisions between $n_{i}$ and $n_{j}$ bubbles in unit volume in time $\Delta t$ is

$$
\Delta G_{i j}=\pi G_{i} G_{j}\left(r_{i}+r_{j}\right)^{2}\left(v_{i}-v_{j}\right) \Delta t,
$$

where the distribution of bubble sizes is given by the function $G(n, t)$ and we have written $G_{i}$ for $G\left(n_{i}, t\right)$. Equation (24) can be written in terms of $n_{i}$ by substituting for $v_{i}$ from Equation (2) and for $r_{i}$ from Equation (9). In terms of the "normalized" distribution function $g(n, t)$, obtained by dividing the function $G(n, t)$ by $m$, the number of gas atoms per unit volume, we obtain, after some simplification,

$$
\Delta g_{i j}=g_{i} g_{j}\left(n_{i}^{1 / 2}+n_{j}^{1 / 2}\right)^{2}\left(n_{i}^{-1 / 2}-n_{j}^{-1 / 2}\right) \Delta \tau^{\prime},
$$

where $\tau^{\prime}=-1.93 \mathrm{ma}_{0} \mathrm{D}_{\mathrm{S}} \mathrm{ft}\left(\gamma_{\mathrm{kT}}\right)^{-1 / 2}$ is a dimensionless "reduced time." (Note that $f$ is negative in this formulation.) Equation (25) is analogous to Equation (17) for the case of random-migration coalescence and has been used in the same manner to obtain the distribution function $g(n, t)$ by finite-difference methods.

\section{B. Approximate Treatment}

It is interesting to consider first an approximate approach similar to those considered previously. Although as stated above the distribution cannot be characterized by a mean radius, it can be characterized by two radii such that

$$
r_{1}=r(1-q)
$$

and

$$
r_{2}=r(1+q)
$$

where $r$ is the mean radius and $q$ is a number less than one, which gives a measure of the variance of the distribution $g(n, t)$. If the distribution is approximated by $N_{1}$ bubbles of radius $r_{1}$ and $N_{2}$ bubbles of radius $r_{2}$, where $N_{1}=N_{2}=\frac{1}{2} N$, then Equation (24) can be simplified to give, for the number of collisions in time $\Delta t$,

$$
\Delta N=-\frac{3.56 \pi a_{0} D_{s} f}{k T} \frac{N^{2} r q}{l-q^{2}} \Delta t
$$

Each collision results in the loss of one bubble of each size and the formation of a larger bubble of radius $r_{3}$. From conservation of gas atoms and Equation (9), $r_{3}$ is given by

$$
r_{3}=\left(r_{1}^{2}+r_{2}^{2}\right)^{1 / 2}=\sqrt{2}\left(1+q^{2}\right)^{1 / 2} r .
$$


Calculation of the new mean radius after a small number of collisions, and subtraction of the original mean radius, gives for the change in mean radius

$$
\Delta \mathrm{r} \cong\left[\sqrt{2}\left(1+\mathrm{q}^{2}\right)^{1 / 2}-1\right](\Delta \mathrm{N} / \mathrm{N}) \mathrm{r} .
$$

Since the concentration of gas atoms $m$ is given by the sum $N_{1} n_{1}+N_{2} n_{2}=$ $\frac{1}{2} \mathrm{~N}\left(\mathrm{n}_{1}+\mathrm{n}_{2}\right)$, it follows from Equation (9) that

$$
N=\frac{3 m k T}{8 \pi r^{2}} \frac{1}{1+q^{2}}
$$

Substituting in Equation (29) for $\Delta \mathrm{N}$ from Equation (28) and for $\mathrm{N}$ from Equation (30), passing to the limit and integrating from $r=r_{0} \cong .0$ at $t=0$ to $r$. at $t$, we obtain, for the mean radius as a function of time,

$$
r \cong-1.34\left[\sqrt{2}\left(1+q^{2}\right)^{1 / 2}-1\right] \frac{q}{1-q^{4}} \frac{m a_{0} D_{s} f t}{\gamma} .
$$

Again the negative sign enters because $f$ is taken negative. It is assumed in the integration that $q$ is independent of $r$. Although the appropriate value of $\mathrm{q}$ is unknown, the more detailed calculations that follow indicate that $q=1 / 2$. This value in Equation (31) gives

$$
r \cong-0.4 \mathrm{ma}_{0} \mathrm{D}_{\mathrm{s}} \mathrm{ft} / \gamma .
$$

It follows from the approximate relation for swelling, as given by Barnes, ${ }^{8}$ that

$$
\Delta \mathrm{V} \cong \frac{\mathrm{mkTr}}{2 \gamma} \cong-0.2(\mathrm{~m} / \gamma)^{2} \mathrm{kTa} \mathrm{a}_{0} \mathrm{ft}
$$

This equation is based on the approximation that all bubbles have radius $r$. If we use instead the better approximation, that half the bubbles have radius $r_{1}$, the other half $r_{2}$, then it can be shown that a correction factor given by $\left(1+3 q^{2}\right) /\left(1+q^{2}\right)$ must be inserted in Equation (3lb). The value of this factor for $q=1 / 2$ is 1.4 , so that the predicted swelling is given by

$$
\Delta \mathrm{V} \cong \frac{1+3 q^{2}}{1+q^{2}} \frac{m k T r}{2 \gamma} \cong-0.28(\mathrm{~m} / \gamma)^{2} k \operatorname{Ta}_{0} \mathrm{D}_{\mathrm{s}} \mathrm{ft}
$$

It will be seen from the results of the next section that this estimate is still about $15 \%$ too low.

C. Determination of the Size-distribution Function

To obtain a more accurate estimate of the behavior of bubbles in a force field, we return to Equation (25) and consider the finite-difference 
approach. The method of calculation is similar to that used in the case of random-migration coalescence. Instead of a singular input, the initial distribution was taken to be the result given by the previous calculation after a very short annealing time. This initial distribution was used becausc the singular input used previously would not permit coalescence,

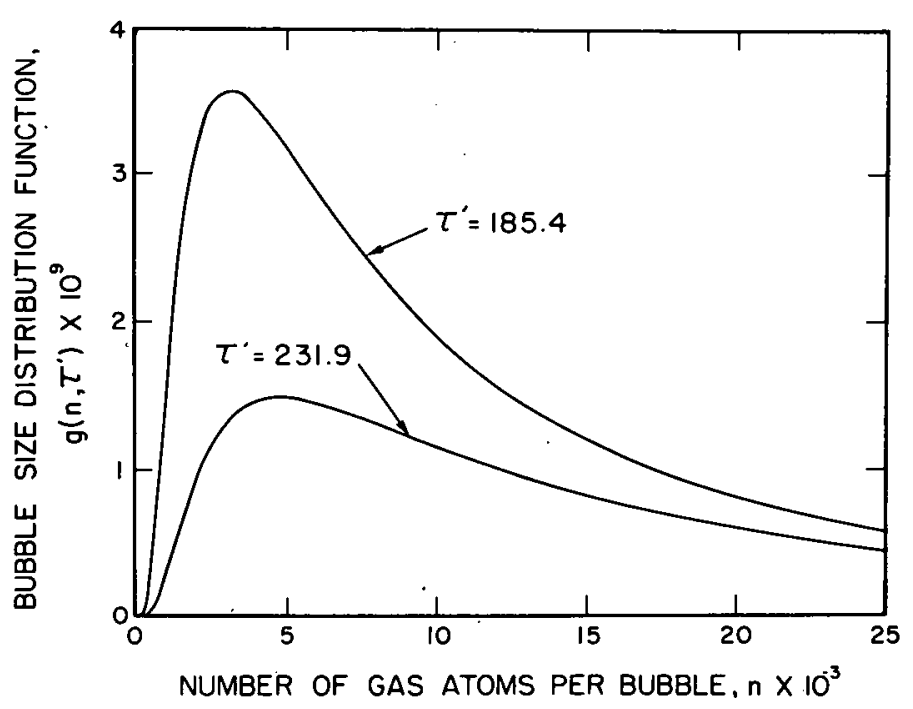

42291

Fig. 5. Size-distribution Functions at Different Times for Biased-migration Bubble Coalescence since Equation (25) predicts no coalescence for equal-sized bubbles; further, some randommigration coalescence would necessarily occur, and initially would dominate the coalescence. Preliminary claculations based on Equation (25) showed that the required $r$ ange in $\mathrm{n}$ increased so rapidly that the doubling procedure used in the previous case was inadequate. The distribution curves given in Fig. 5 indicate that the distribution at any time $t$ decreases very slowly with increasing $\mathrm{n}$. It was noted, however, that plotting $g\left(n, \tau^{\prime}\right)$ vs $\log n$ yielded a curve resembling a normal distribution (Fig. 6). This curve

decreases fairly sharply to zero at both large and small values of log $\mathrm{n}$. Because of this behavior $n_{i}$ was taken as $\exp (i / 10)$, and $i$ was taken from 1 to 125 . This approach uses value $s$ of $\mathrm{n}_{i}$ that are uniformly spaced on a logarithmic scale. To increase the range in $n$, it was necessary only to add values at the high end; when this became necessary, it was possible to drop values at the low end because $g\left(n, \tau^{\prime}\right)$ was es sentially zero for the small values of $\mathrm{n}$.

This approach leads to some difficulty and inaccuracy because of the problem of assigning the correct subscript to the bubbles formed by coalescence. The method used was to take "differentials,"

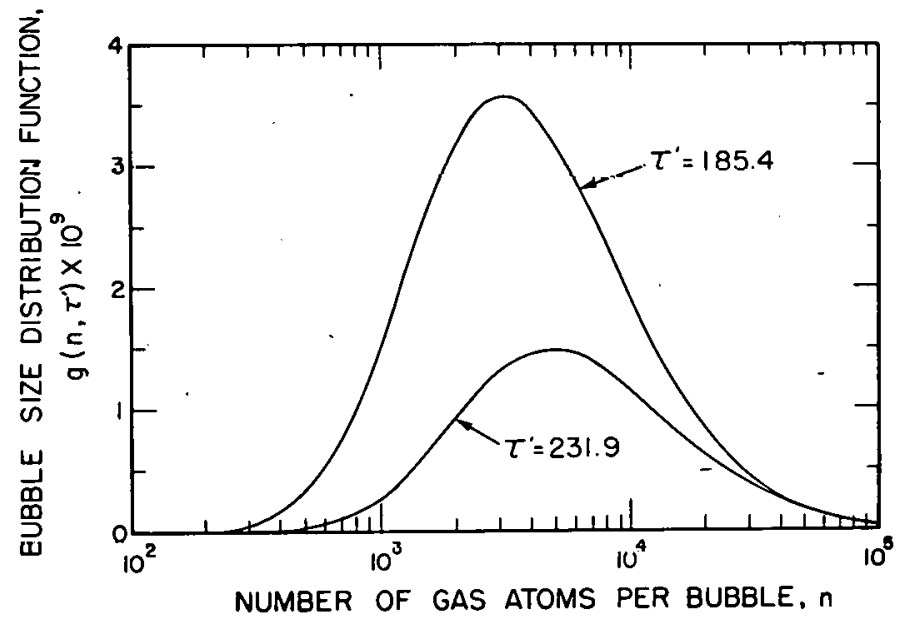

42290

Fig. 6. Semilogarithmic Plot of Distribution Function for Biased-migration Coalescence 


$$
\Delta \mathrm{n}_{\mathrm{i}}=\mathrm{n}_{\mathrm{i}} / 10
$$

which follows from the definition of $n_{i}$ as an exponential, and to pair the se increments with the appropriate values of $n_{i}$. Each time $i$ and. $j$ bubbles were compared, the appropriate value of $\mathrm{k}$ for the newly formed bubble was calculated. Slight inaccuracy enters in this approach because $i, j$, and $\mathrm{k}$ denote a range in $\mathrm{n}$, and not discrete values. The calculations were then carried out as in the previous case.

The moments and characteristic values calculated for the preceding case were also calculated for this case. Equation (20) was slightly modified, since in this case $\Delta n$ is a function of $i$, and only 125 values of $i$ were considered.

D. Results

The calculated distributions in this case were extremely smooth, with no oscillation as detected in the preceding case, although the results were not as accurate. The calculated number of atoms, which should be constant, varied from $m$ through a maximum of $1.11 \mathrm{~m}$ at $\tau^{\prime}=8.5$, then decreased slowly to $1.014 \mathrm{~m}$ at $\tau^{\prime}=568$, the highest value considered. The number of time steps used was 1300 , which is considerably more than in the previous case.

The results are generally consistent with the approximate calculation given above. That is, the predicted mean radius and volume change are very nearly linear with time, and the characteristic values of $n$ are proportional

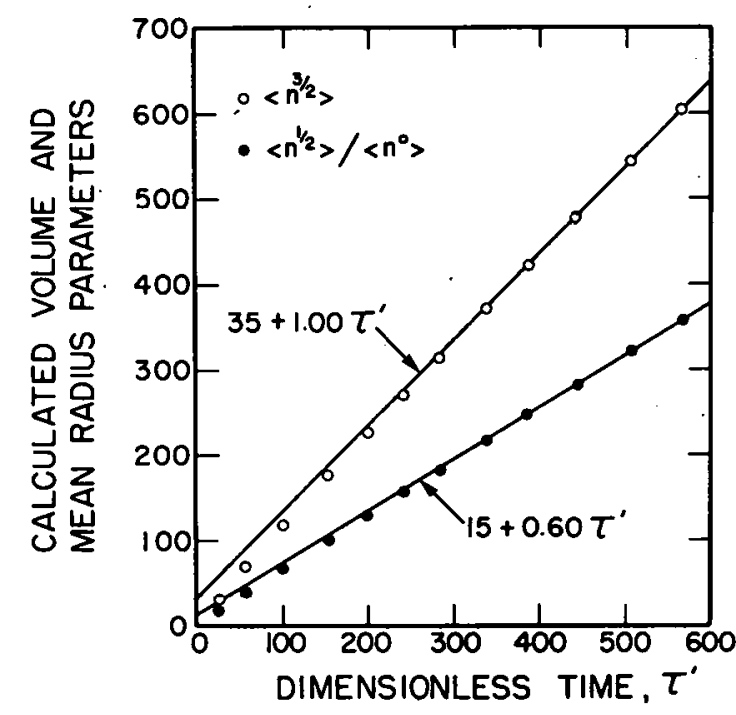

42289

Fig. 7. Dependence of Bubble-distribution Parameters on Timc. The mean radius $r$ is a function of the ratio of moments $\left\langle n^{1 / 2}\right\rangle /\left\langle n^{\circ}\right\rangle$, and the swelling $\Delta v$ is a function of the moment $\left\langle n^{3 / 2}\right\rangle$. to the square of $\tau^{\prime}$. At $\tau^{\prime}=568$, the calculated slopes are 0.977 for the mean radius and 0.945 for the volume change. The difference results primarily from the error in $m$. This error affects the mean radius calculation less because the mean radius is given by the ratio of two moments [see Equation.(13)], so that the error in $m$ is effectively cancelled. The results are given in Fig. 7 , in which the ratio of moments $\left\langle n^{1 / 2}\right\rangle /\left\langle n^{0}\right\rangle$ and the moment $\left\langle\mathrm{n}^{3 / 2}\right\rangle$ are plotted as func tions of $T^{\prime}$. These results can be expressed approximately by the equations

$\left\langle\mathrm{n}^{1 / 2}\right\rangle /\left\langle\mathrm{n}^{0}\right\rangle=15+0.60 \tau^{1}$

and

$(1 / m)\left\langle n^{3}\right\rangle=35+1.00 \tau^{\prime}$,

which correspond to the lines drawn in Fig. 7 . 
The calculated slopes for the characteristic values of $\mathrm{n}$ are approximately 1.94. If, as in the preceding case, it is assumed that the distribution approaches a standard shape, with decaying amplitude and spreading range, then it can be shown from the above results that the distribution must be of the form

$$
G(n, t)=m \tau^{1-4} Z_{2}\left(u^{\prime}\right)
$$

where $u^{\prime}=n / T^{\prime 2}$. Calculations of the standard frequency curve $Z_{2}\left(u^{\prime}\right)$ for several values of $\tau^{\prime}$ give the results summarized in Fig. 8. It appears that the distribution tends toward a standard shape with increasing time, but not nearly as rapidly as in the preceding case.

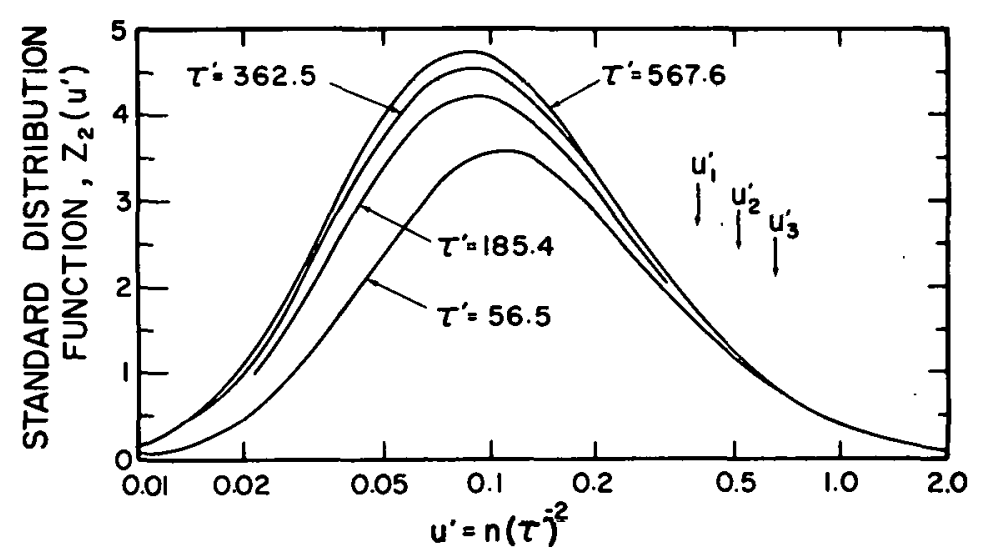

42288

Fig. 8. "Standardized" Size-distribution Curves for Biased-migration Coalescence

The predicted values for the mean radius and volume change, from Equations (13), (15), (24), and (31), are

$$
\mathrm{r} \cong-0.40 \mathrm{ma}_{0} \mathrm{D}_{\mathrm{C}} \mathrm{ft.} / \gamma
$$

and

$$
\Delta V \cong-0.33 \mathrm{~m}^{2} \mathrm{kT} \mathrm{a}_{0} \mathrm{D}_{\mathrm{s}} \mathrm{ft} / \gamma^{2}
$$

where the constant terms in Equation (32) have been omitted. Comparison of Equations (34a) and (30) leads to the conclusion mentioned previously that $\mathrm{q}=1 / 2$. Although this value of $\mathrm{q}$ brings the approximate results intoagreement for the mean radius, the error in $\Delta V$ is still significant $[\sim 65 \%$ compared to Equation ( $3 \mathrm{lb})$ ] because of the relatively wide variation in bubble. size compared to the previous case.

The force on the atoms can be related to a surface-diffusion heat of transport, $Q_{S}^{*}$, by comparing the flux relation used in the derivation of 
Equation (1) to the flux relation given by Denbigh ${ }^{12}$ for diffusion in a thermal gradient. The surface density $\nu$ is considered constant, so that the result is ${ }^{3}$

$$
f=-\frac{Q_{S}^{*} \nabla T}{T}
$$

Although no experimental data are available for the heat of transport for surface diffusion, an order-of-magnitude estimate of $Q_{S}^{*} \sim 10 \mathrm{kcal} / \mathrm{mole}$ will suffice to give a semiquantitative measure of the results of coalescence to be expected according, to the present theory. For a thermal gradient of $10^{3}{ }^{\circ} \mathrm{C} / \mathrm{cm}$, the results for copper at $1000^{\circ} \mathrm{K}$ with $\mathrm{m}=10^{20}$ atoms $/ \mathrm{cm}^{3}$ are, from Equations (34a) and (34b),

$$
\frac{\mathrm{r}}{\mathrm{t}} \cong 0.4 \AA \mathrm{A} / \mathrm{sec},
$$

and

$$
\frac{\Delta V}{t} \cong 2.7 \times 10^{-3} \% / \mathrm{sec}
$$

The results for an annealing time of $\mathrm{l}$ hr are $\mathrm{r} \approx 1400 \AA$ and $\Delta \mathrm{V} \approx 10 \%$. The effects of changing $\mathrm{m}, \mathrm{T}$, or $\nabla \mathrm{T}$ are apparent from Equations (34) and (35).

\section{DISCUSSION OF RESULTS}

Equations have been derived for the speed of pore migration in an energy gradient and for pore diffusivity under the assumption that a small pore migrates by surface diffusion. Other migration mechanisms, such as evaporation and condensation at the leading and trailing surfaces, ${ }^{13}$ may be expected to be significant in larger bubbles.

The resulting equations, which generally confirm earlier and usually more approximate results, have been applied in analyses of bubble coalescence upon postirradiation annealing for two highly idealized cases. In these two cases (coalescence resulting from random migration and from biased migration in an energy gradient), several as sumptions and approximations have been made. The basic assumptions are that the bubbles contain ideal gas at a pressure balanced by the surface tension of the solid, that they are present initially as very small, randomly distributed bubbles in a perfect crystal, and that coalescence occurs instantaneously when two bubbles meet. We have ignored the problems of bubble nucleation, interactions with dislocations or grain boundaries, migration by other mechanisms, and equilibration of the gas pressure and surface tension after coalescence, although many of these considerations have heen discussed previously. $8,14,15$ 
Despite these limitations, several results are significant. First, swelling is not likely to be significant if it occurs only by random migration and coalescence of bubbles in large-grained material, but swelling can be greatly enhanced if some external effect can influence bubble migration. A similar conclúsion was reached by Loomis and Pracht; recrystallization was shown to be a necessary prerequisite for pronounced swelling in their experimental investigation of alpha uranium. ${ }^{10}$

Second, the importance of considering the entire distribution of bubble sizes, rather than only the mean radius, has been emphasized, and it has been shown that the mean bubble size gives only a semiquantitative measure of the swelling.

In addition, the fact that the calculated distributions approach a standard form should be very useful in future work, for it implies that the approximate relation for swelling in terms of the mean radius [Equation $(31 b)$ ] is correct except for a proportionality constant. The assumption of a standard distribution may also lead to simpler treatments of problems such as that treated here. Such treatments would be very useful in consideration of other problems, such as coalescence of bubbles on dislocations ${ }^{9}$ or grain boundaries.

In conclusion, it should be noted that the finite-difference method developed here is a powerful method that can be applied not only to more realistic cases of bubble coalescence, but to a variety of other problems, such as colloid coagulation. ${ }^{7}$

\section{ACKNOWLEDGMENTS}

Several helpful discussions with members of the Metallurgy Division and with P. G. Shewrnon are gratefully acknowledged. 
1. R. S. Barnes and D. J. Mazey, Proc. Roy. Soc. 275 (1963), 47.

2. R. S. Nelson, D. J. Mazey, and R. S. Barnes, private communication.

3. P. G. Shewmon, Trans AIME 230 (1964), 1134.

4. F. A. Nichols, to be published in J. Appl. Phys.

5. W. Jost, "Diffusion in Solids, Liquids, Gases," Academic Press, Inc., New York: (1952).

6. G. W. Greenwood and M. V. Speight, J. Nucl. Mat. 10 (1963) 140.

7. S. Chandrasekhar, Rev. Mod. Phys. 15 (1943), 1.

8. R. S. Barnes, J. Nucl. Mat. 11 (1964), 135.

9. M. V. Speight, J. Nucl. Mat. 12 (1964), 216.

10. B. A. Loomis and D. W. Pracht, J. Nucl. Mat. 10 (1963), 346.

11. M. V. Speight and G. W. Greenwood, Phil. Mag. 9' (1964), 683.

12. K. G. Denbigh, The Thermodynamics of the Steady State, John Wiley and Sons, Inc., New.York (195l).

13. M. V. Speight, J. Nucl. Mat. 13 (1964), 207.

14. G. W. Greenwood, A. J. E. Foreman, and D. E. Rimmer, J. Nucl. Mat. 4 (1959), 305.

15. J. A. Brinkman, Nucl. Met. AIME, IMD 6 (1959), 1. 